TSUKUBA J. MATH.

Vol. 12 No. 2 (1988), 489-495

\title{
HOMEOMORPHISMS OF ZERO-DIMENSIONAL SPACES
}

\author{
By \\ Takashi KIMURA \\ Dedicated to Professor Yukihiro Kodama on his 60th birthday
}

\section{Introduction.}

All spaces considered in this paper are assumed to be compact and metrizable.

Let $\varphi$ be a homeomorphism from a space $(X, d)$ onto itself. Then $\varphi$ is expansive if there is $c>0$ such that for every $x, y \in X$ with $x \neq y$ there is $n \in \boldsymbol{Z}$ for which $d\left(\varphi^{n}(x), \varphi^{n}(y)\right)>c$. Given $\delta>0$, a sequence $\left\{x_{i}: i \in \boldsymbol{Z}\right\}$ is a $\delta$-pseudoorbit of $\varphi$ if $d\left(\varphi\left(x_{i}\right), x_{i+1}\right)<\delta$ for every $i \in \boldsymbol{Z}$. Given $\varepsilon>0$, a sequence $\left\{x_{i}: i \in \boldsymbol{Z}\right\}$ is $\varepsilon$-traced by a point $y \in X$ if $d\left(\varphi^{i}(y), x_{i}\right)<\varepsilon$ for every $i \in \boldsymbol{Z}$. We say that $\varphi$ has the pseudo orbit tracing property (abbrev. P.O.T.P.) if for every $\varepsilon>0$ there is $\delta>0$ such that every $\delta$-pseudo-orbit of $\varphi$ can be $\varepsilon$-traced by some point of $X$.

For a space $(X, d)$ we denote by $\mathcal{K}(X)$ the space of all homeomorphisms of $X$ with the metric $d(\varphi, \phi)=\max \{d(\varphi(x), \phi(x)): x \in X\}$ for every $\varphi, \phi \in \mathcal{K}(X)$. Let $\mathcal{E}(X)=\{\varphi \in \mathcal{H}(X): \varphi$ is expansive $\}$ and $\mathscr{P}(X)=\{\varphi \in \mathscr{H}(X): \varphi$ has P.O.T.P. .

In Section 3 we are concerned with the Cantor set $C$. The Cantor set $C$ is the unique zero-dimensional infinite group. N. Aoki [1] proved that every group automorphism of $C$ has P.O.T.P. M. Sears [6] proved that $\mathcal{E}(C)$ is dense in $\mathscr{H}(C)$, constructing a dense subset $\mathcal{A}$ of $\mathcal{E}(C)$ in $\mathscr{H}(C)$. M. Dateyama [3] proved that $\mathscr{P}(C)$ is dense in $\mathscr{H}(C)$, constructing a dense subset $\mathscr{B}$ of $\mathscr{P}(C)$ in $\mathscr{H}(C)$. However, for the sets $\mathcal{A}$ and $\mathscr{B}$ above we have $\mathcal{A} \cap \mathscr{B}=\phi$. So it is unknown whether the set $\mathcal{E}(C) \cap \mathscr{P}(C)$ of all expansive homeomorphisms with P.O.T.P. of $C$ is dense in $\mathscr{H}(C)$. In Section 3 we shall prove the following theorem.

THEOREM 1. The set of all expansive homeomorphisms with P. O. T.P. of the Cantor set $C$ is dense in $\mathscr{H}(C)$.

We know [6] that $\mathcal{E}(C)$ is of first category. So $\mathcal{E}(C) \cap \mathscr{P}(C)$ is also of first category.

The convergent sequence is another standard zero-dimensional space, classed with the Cantor set. In Section 4 we shall prove the following theorem.

Received October 22, 1987 
TheOREM 2. Let $S=\{0,1,1 / 2,1 / 3, \cdots\}$. Then

(a) the set of all expansive homeomorphisms of $S$ is dense in $\mathscr{H}(S)$,

(b) the set of all homeomorphisms with P.O.T.P. of $S$ is dense in $\mathscr{H}(S)$,

(c) $S$ has no expansive homeomorphism with P.O.T.P.

In Section 5 we shall construct a zero-dimensional space having no expansive homeomorphism.

\section{Preliminaries.}

Let $D^{\boldsymbol{Z}}=\Pi\left\{D_{i}: i \in \boldsymbol{Z}\right\}$, where $D_{i}=\{0,1\}$ for every $i \in \boldsymbol{Z}$. We define the metric $d$ on $D^{z}$ by

$$
d(x, y)=\left\{\begin{array}{lll}
1 / \min \left\{|k|: x_{k} \neq y_{k}\right\} & \text { if } & x_{0}=y_{0} \\
2 & \text { if } & x_{0} \neq y_{0}
\end{array}\right.
$$

for every $x=\left(x_{i}\right), y=\left(y_{i}\right) \in D^{z}$.

Obviously, $\left(D^{z}, d\right)$ is homeomorphic to the Cantor set. For a homeomorphism of a compact metrizable space $X$ it is clear that both expansiveness and P.O.T.P. do not depend on the choice of metrics on $X$. Thus we may regard $\left(D^{z}, d\right)$ as the Cantor set.

For every $i, j \in \boldsymbol{Z}$ with $i \leqq j$ we put $D(i, j)=\Pi\left\{D_{k}: i \leqq k \leqq j\right\}$ and for every $f \in D(i, j)$ we put $c^{+}(f)=j$ and $c^{-}(f)=i$. We define the order $\leqq$ on $\cup\{D(i, j): i, j$ $\in \boldsymbol{Z}$ with $i \leqq j\} \cup D^{\boldsymbol{Z}}$ as follows : $f \leqq g$ if and only if one of the following conditions holds; (1) $f=g$, (2) $f \in D(i, j), g \in D(k, l), k \leqq i, j \leqq l$ and $f_{m}=g_{m}$ for every $m, i \leqq m \leqq j$, (3) $f \in D(i, j), g \in D^{z}$ and $f_{m}=g_{m}$ for every $m, i \leqq m \leqq j$, where $f=$ $\left(f_{i}, f_{i+1}, \cdots, f_{j}\right)$ for $f \in D(i, j)$ and $f=\left(\cdots, f_{-1}, f_{0}, f_{1}, \cdots\right)$ for $f \in D^{z}$. For every $f \in D(i, j)$ and any $n \in \boldsymbol{N}$ with $i \leqq-n$ and $n \leqq j$ (or for every $f \in D^{\boldsymbol{Z}}$ and any $n \in \boldsymbol{N})$ we put $f_{1 n}=\left(f_{-n}, f_{-n+1}, \cdots, f_{n}\right) \in D(-n, n)$. For every $f \in D(i, j)$ we put $A_{f}=p_{i j}^{-1}(f)$, where $p_{i j}: D^{z} \rightarrow D(i, j)$ is the projection.

If a space $X$ is the union of a pairwise disjoint collection $\left\{X_{\lambda}: \lambda \in \Lambda\right\}$ of open-and-closed subsets of $X$, then we represent $X$ as $X=\bigoplus\left\{X_{\lambda}: \lambda \in \Lambda\right\}$.

\section{Proof of Theorem 1.}

Let $\phi: D^{z} \rightarrow D^{z}$ be a homeomorphism and $\varepsilon>0$. We shall construct an expansive homeomorphism $\varphi$ with P. O. T. P. such that $d(\psi, \varphi)=\max \{d(\psi(x), \varphi(x))$ : $\left.x \in D^{Z}\right\}<\varepsilon$.

We take $k, n \in \boldsymbol{N}$ such that $1 / k<\varepsilon$ and $d(\phi(x), \phi(y))<1 / k$ for every $x, y \in D^{\boldsymbol{Z}}$ with $d(x, y)<1 / n$. 
Claim 1. For every $f \in D(-n, n)$ there are $h(f) \in D(-k, k)$ and $g(f) \in$ $D\left(-l_{1}, l_{2}\right)$ for some $l_{1}, l_{2} \in N, i=1,2$, satisfying the following three conditions;

(a) $D^{z}=\oplus\left\{A_{g(f)}: f \in D(-n, n)\right\}$,

(b) $\psi\left(A_{f}\right) \subset A_{h(f)}$,

(c) $h(f) \leqq g(f)$.

Proof of Claim 1. From $\operatorname{diam} A_{f}<1 / n$ it follows that $\operatorname{diam} \phi\left(A_{f}\right)<1 / k$. Since $D^{z}=\oplus\left\{A_{h}: h \in D(-k, k)\right\}$ and $d\left(A_{h}, A_{h^{\prime}}\right) \geqq 1 / n$ for every $h, h^{\prime} \in D(-k, k)$ with $h \neq h^{\prime}$, there is $h(f) \in D(-k, k)$ such that $\phi\left(A_{f}\right) \subset A_{h(f)}$. For every $h \in$ $D(-k, k)$ list $\{f \in D(-n, n): h(f)=h\}$ as $\left\{f_{h i}: 1 \leqq i \leqq p_{h}\right\}$. For every $i, 1 \leqq i \leqq p_{h}$, we take $g_{h i} \geqq h$ such that $A_{h}=\bigoplus\left\{A_{g_{h i}}: 1 \leqq i \leqq p_{h}\right\}$. Let us set $g\left(f_{h i}\right)=g_{h i}$ for every $h \in D(-k, k)$ and any $i, 1 \leqq i \leqq p_{h}$. Then $g(f)$ and $h(f)$ have all the required properties.

Next, we shall construct a homeomorphism $\varphi: D^{z} \rightarrow D^{z}$. For every $x \in D^{z}$ we define $\varphi(x)$ as follows.

Let $f=x_{1 n} \in D(-n, n)$ and $g(f) \in D\left(-l_{1}, l_{2}\right)$.

Case $1 . \quad l_{1}+l_{2} \geqq 2 n$ and $l_{2} \geqq n$.

Let us set

and

$$
(\varphi(x))_{i}=\left\{\begin{array}{lll}
(g(f))_{i} & \text { if }-l_{1} \leqq i \leqq l_{2} \\
x_{i+1} & \text { if } \quad l_{2}+1 \leqq i \\
x_{i+l_{1}+l_{2}+2} & \text { if } n-l_{1}-l_{2}-1 \leqq i \leqq-l_{1}-1 \\
x_{i-2 n+l_{1}+l_{2}+1} & \text { if } & i \leqq n-l_{1}-l_{2}-2
\end{array}\right.
$$

$$
M^{+}(f)=1 \quad \text { and } \quad M^{-}(f)=-2 n+l_{1}+l_{2}+1 .
$$

Case 2. $l_{1}+l_{2}<2 n$ and $l_{1} \leqq n$.

Let us set

and

$$
(\varphi(x))_{i}= \begin{cases}(g(f))_{i} & \text { if }-l_{1} \leqq i \leqq l_{2} \\ x_{i+1} & \text { if } i \leqq-n-2 \\ x_{i+2 n+2} & \text { if }-n-1 \leqq i \leqq-l_{1}-1 \\ x_{i+2 n-l_{1}-l_{2}+1} & \text { if } l_{1}+1 \leqq i\end{cases}
$$

$$
M^{+}(f)=2 n-l_{1}-l_{2}+1 \text { and } M^{-}(f)=1 .
$$

Case 3. otherwise, i. e. $\left(l_{1}+l_{2} \geqq 2 n\right.$ and $\left.l_{2}<n\right)$ or $\left(l_{1}+l_{2}<2 n\right.$ and $\left.l_{1}>n\right)$.

In this case we have $l_{2}<n<l_{1}$. Let us set 
and

$$
(\varphi(x))_{i}=\left\{\begin{array}{lll}
(g(f))_{i} & \text { if } & -l_{1} \leqq i \leqq l_{2} \\
x_{i+n-l_{2}} & \text { if } & l_{2}+1 \leqq i \\
x_{i+l_{1}-n} & \text { if } & i \leqq-l_{1}-1
\end{array}\right.
$$

$$
M^{+}(f)=n-l_{1} \quad \text { and } \quad N^{-}(f)=l_{1}-n .
$$

Then it is obvious that $\varphi_{\mid A_{f}}: A_{f} \rightarrow A_{g(f)}$ is a homeomorphism. By (a), $\varphi$ is a homeomorphism from $D^{\boldsymbol{Z}}$ onto itself. Let us set $m=\max \left\{-c^{-}(g(f)), c^{+}(g(f))\right.$ : $f \in D(-n, n)\}$.

By the construction of $\varphi$ the following claim is easily seen.

Claim 2. Let $x, y \in D^{z}$ with $d(x, y)=1 / k \leqq 1 / 2 m$.

(i) If $x_{k} \neq y_{k}$, then $d(\varphi(x), \varphi(x))=1 / l$ and $x_{l} \neq y_{l}$, where $l=k-M^{+}\left(x_{1 n}\right)$.

(ii) If $x_{-k} \neq y_{-k}$, then $d\left(\varphi^{-1}(x), \varphi^{-1}(y)\right)=1 / l$ and $x_{-l} \neq y_{-l}$, where $l=k-$ $M^{-}\left(x_{\mid n}\right)$.

By Claim 2, $1 / 2 m$ is an expansive constant for $\varphi$. Thus $\varphi$ is expansive.

To prove that $\varphi$ has P.O.T.P. we need the following mappings $\alpha$ and $\beta$.

For every $f \in \cup\{D(i, j): i, j \in \boldsymbol{Z}$ with $i \leqq-n$ and $n \leqq j\}$ let us set

$$
\alpha(f)=\max \left\{g: g<\varphi(h) \text { for every } h \in D^{Z} \text { with } f<h\right\} .
$$

For every $g \in \cup\{D(i, j): i, j \in \boldsymbol{Z}$ with $i \leqq-m$ and $m \leqq j\}$ let us set

$$
\beta(g)=\max \left\{f: f<\varphi^{-1}(h) \text { for every } h \in D^{Z} \text { with } g<h\right\} .
$$

We shall show that $\varphi$ has P.O.T.P.

Let $\varepsilon_{1}>0$. We take $\delta=1 / N$ such that $1 / N<\min \left\{\varepsilon_{1}, 1 / 2 m\right\}$. Let $\left\{x^{i}: i \in \boldsymbol{Z}\right\}$ be a $\delta$-pseudo-orbit of $\varphi$. Let $K(-1)=-N-1$. By induction on $0 \leqq i \in Z$, we choose $K(i)$ and $y_{j} \in D_{j}$ for every $j, K(i-1)<j \leqq K(i)$, satisfying the following conditions :

(d) $K(i-1)<K(i)$,

(e) $c^{+}\left(\alpha^{i}\left(y^{i}\right)\right)=N$,

(f) $\alpha^{i}\left(y^{i}\right)_{\mid N}=x_{1 N}^{k}$, where $y^{i}=\left(y_{-N}, y_{-N+1}, \cdots, y_{K(i)}\right) \in D(-N, K(i))$.

In case $i=0$, let $K(0)=N$ and for every $j,-K(-1)<j \leqq K(0)$, let $y_{j}=x_{j}^{0}$. Assume that $K(i)$ and $y_{j}, K(i-1)<j \leqq K(i)$, are chosen such that the above conditions hold. Let us set $K(i+1)=K(i)+M^{+}\left(\alpha^{i}\left(y^{i}\right)_{\mid n}\right)$ and $y_{j}=x_{j+N-K(i+1)}^{i+1}$ for every $j, K(i)<j \leqq K(i+1)$. It is easy to check that all induction hypothesis are satisfied. Let $L(1)=N+1$. By induction on $0 \geqq i \in \boldsymbol{Z}$, similarly as above, we choose $L(i)$ and $y_{j} \in D_{j}$ for every $j, L(i) \leqq j<L(i+1)$, satisfying the following conditions : 
(g) $L(i)<L(i+1)$,

(h) $c^{-}\left(\beta^{-i}\left(y^{i}\right)\right)=-N$,

(i) $\beta^{-i}\left(y^{i}\right)_{1 N}=x_{1 N}^{k}$,

where $y^{i}=\left(y_{L(i)}, y_{L(i)+1}, \cdots, y_{N}\right) \in D(L(i), N)$. Let us set $y=\left(\cdots, y_{-1}, y_{0}, y_{1}, \cdots\right)$ $\in D^{z}$. Then for every $i \geqq 0$ we have $\varphi^{i}(y)>\alpha^{i}\left(y^{i}\right)$ and $\alpha^{i}\left(y^{i}\right)_{\mid N}=x_{\mid N}^{i}$. This implies that $\varphi^{i}(y)_{\mid N}=x_{1 N}^{i}$ and therefore we have $d\left(\varphi^{i}(y), x^{i}\right)<1 / N<\varepsilon_{1}$. For every $i \leqq 0$ we have $\varphi^{i}(y)>\beta^{-i}\left(y^{i}\right)$ and $\beta^{-i}\left(y^{i}\right)_{\mid N}=x_{{ }_{N}}^{i}$. This implies that $\varphi^{i}(y)_{\mid N}=x_{{ }_{N}}^{i}$ and therefore we have $d\left(\varphi^{i}(y), x^{i}\right)<1 / N<\varepsilon_{1}$. Hence $\left\{x^{i}: i \in \boldsymbol{Z}\right\}$ is $\varepsilon_{1}$-traced by $y$. Therefore $\varphi$ has P.O.T.P.

We show that $d(\varphi, \psi)<\varepsilon$. By the construction of $\varphi, \varphi\left(\left(A_{f}\right)\right)=A_{g(f)}$ for every $f \in D(-n, n)$. For every $x \in D^{z}$, we have $x \in A_{f}$ for some $f \in D(-n, n)$. Thus, by (c), we have $\varphi(x) \in \varphi\left(A_{f}\right)=A_{g(f)} \subset A_{h(f)}$. On the other hand, by (b), we have $\phi(x) \in \phi\left(A_{f}\right) \subset A_{h(f)}$. From $\operatorname{diam} A_{h(f)}=1 /(k+1)<\varepsilon$ it follows that $d(\varphi(x), \psi(x))$ $<\varepsilon$. Hence we have $d(\varphi, \phi)<\varepsilon$. Theorem 1 has been proved.

\section{Proof of Theorem 2.}

Let $d$ be the Euclidean metric on $S=\{0,1,1 / 2,1 / 3, \cdots\}$. Note that a mapping $\varphi: S \rightarrow S$ is a homeomorphism if and only if $\varphi$ is one-to-one, onto and $\varphi(0)=0$. For every $n \in N$ we set $S_{n}=\{1 /(n-1), 1 /(n-2), \cdots, 1\}$.

(a) Let $\phi \in \mathscr{H}(S)$ and $\varepsilon_{0}>0$. We construct $\varphi \in \mathcal{E}(S)$ such that $\tilde{d}(\varphi, \phi)<\varepsilon_{0}$. To do this, we take $n \in \boldsymbol{N}$ with $1 / n<\varepsilon_{0}$. For every $m \in \boldsymbol{N}, m<n$, we take $x_{m} \in S$ such that $\phi\left(x_{m}\right)=1 / m$. Let $l=\max \left\{1 / x_{m}: m<n\right\}+1$. For every $k \in \boldsymbol{N}$, $k \geqq l$, let us set

$$
\varphi(1 / k)= \begin{cases}1 /(k-2) & \text { if } k=l+2 i \text { for some } i \in N \\ 1 /(k+2) & \text { if } k=l+2 i-1 \text { for some } i \in N \\ 1 /(l+1) & \text { if } k=l .\end{cases}
$$

For every $m \in \boldsymbol{N}, m<n$, let us set $\varphi\left(x_{m}\right)=1 / m\left(=\phi\left(x_{m}\right)\right)$. Let $\varphi(0)=0$, and for every $x \in S_{l}-\left\{x_{m}: m<n\right\}$ let $\varphi(x)$ be an element of $S_{l}-S_{n}$ such that $\varphi(x) \neq \varphi\left(x^{\prime}\right)$ for every $x, x^{\prime} \in S_{l}-\left\{x_{m}: m<n\right\}$ with $x \neq x^{\prime}$. Then $\varphi$ is one-to-one, onto and $\varphi(0)=0$. Thus $\varphi \in \mathcal{H}(S)$. By the construction of $\varphi$, it is obvious that $d(\varphi, \phi) \leqq$ $1 / n<\varepsilon_{0}$. Let $c=1 /\left(2 l^{2}+2 l\right)$. Note that $U_{c}(1 / l)=\{1 / l\}$. We show that $c$ is an expansive constant for $\varphi$. Let $x, y \in S$ with $x \neq y$. We may assume that $x \neq 0$. If $x \in S_{l}$, then $d(x, y)>c$. If $x \notin S_{l}$, then $\varphi^{i}(x)=1 / l$ for some $i \in Z$, and therefore $d\left(\varphi^{i}(x), \varphi^{i}(y)\right)>c$. Hence we have $\varphi \in \mathcal{E}(S)$.

(b) Let $\phi \in \mathscr{H}(S)$ and $\varepsilon_{0}>0$. We costruct $\varphi \in \mathscr{Q}(S)$ such that $d(\varphi, \phi)<\varepsilon_{0}$. Let $n, l$ and $x_{m}, m<n$, be as in (a). For every $x \in S_{l}$ let $\varphi(x)$ be as in (a). 
For every $x \in S-S$, let $\varphi(x)=x$. Then, similarly as in (a), we have $\varphi \in \mathscr{H}(S)$ and $d(\varphi, \phi)<\varepsilon_{0}$. To prove that $\varphi$ has P.O.T.P. let $\varepsilon_{1}>0$. Take $k \in \boldsymbol{N}$ with $1 / k<\min \left\{\varepsilon_{1}, 1 / l\right\}$. Let $\delta=1 /\left(k^{2}+k\right)$. Note that $U_{\delta}(1 / j)=\{1 / j\}$ for every $j \in \boldsymbol{N}$, $j \leqq k$. It suffices that every $\delta$-pseudo-orbit of $\varphi$ can be $\varepsilon_{1}$-traced by some point of $S$. Let $\left\{y_{i}: i \in Z\right\}$ be a $\delta$-pseudo-orbit of $\varphi$. If $y_{0} \in S-S_{k}$, then $y_{i} \leqq 1 / n<\varepsilon_{1}$ for every $i \in \boldsymbol{Z}$. Thus $\left\{y_{i}: i \in \boldsymbol{Z}\right\}$ is $\varepsilon_{1}$-traced by $y_{0}$. If $y_{0} \in S_{k}$, then $y_{i}=\varphi^{i}\left(y_{0}\right)$ for every $i \in \boldsymbol{Z}$. Thus $\left\{y_{i}: i \in \boldsymbol{Z}\right\}$ is $\varepsilon_{1}$-traced by $y_{0}$. Hence $\varphi$ has P.O.T.P.

(c) Let $\varphi \in \mathcal{E}(S)$ with an expansive constant $c$. It is enough to prove that $\varphi \notin \mathscr{P}(S)$. We take $n \in \boldsymbol{N}$ with $1 / n<c$. Assume that $1 / m$ is a periodic point for every $m \in \boldsymbol{N}, m<n$. Then $\cup\{\operatorname{Orb}(1 / m): m<n\}$ is finite, where $\operatorname{Orb}(x)=$ $\left\{\varphi^{i}(x): i \in \boldsymbol{Z}\right\}$. Pick up a point $x \in S-(\cup\{\operatorname{Orb}(1 / m): m<n\} \cup\{0\})$. Then we have $\operatorname{Orb}(x) \subset S-S_{n}$, therefore $d\left(\varphi^{i}(x), \varphi^{i}(0)\right) \leqq 1 / n<c$ for every $i \in \boldsymbol{Z}$. This is a contradiction. Take $m<n$ such that $1 / m$ is not a periodic point. Let $\varepsilon=$ $1 /\left(m^{2}+m\right)$. For every $\delta>0$ we can take $l \in N$ such that $\varphi^{l-1}(1 / m)<\delta$ and $\varphi^{-l}(1 / m)<\delta$, because $\lim _{i \rightarrow \infty} \varphi^{i}(1 / m)=0$ the $\lim _{i \rightarrow \infty} \varphi^{-i}(1 / m)=0$. Let us set

$$
y_{2 k l+j}=\left\{\begin{array}{lll}
\varphi^{j}(1 / m) & \text { if } & 0 \leqq j \leqq l-1 \\
\varphi^{j-2 l}(1 / m) & \text { if } & l \leqq j \leqq 2 l
\end{array}\right.
$$

Then $\left\{y_{i}: i \in \boldsymbol{Z}\right\}$ is a $\delta$-pseudo-orbit of $\varphi$. Assume that $\left\{y_{i}: i \in \boldsymbol{Z}\right\}$ is $\varepsilon$-traced by $y \in S$. Since $U_{\delta}(1 / m)=\{1 / m\}$ and $y_{2 k l}=1 / m$ for every $k \in \boldsymbol{Z}$, we have $\varphi^{2 k l}(y)$ $=1 / m$ for every $k \in \boldsymbol{Z}$. This implies that $1 / m$ is a periodic point. This is a contradiction. Hence $S$ has no expansive homeomorphism with P.O.T.P.

\section{A zero-dimensional space having no expansive homeomorphism.}

S. Fujii [4] proved that a space $X$ is zero-dimensional if and only if the identity mapping $\mathrm{id}_{X}$ has P.O.T.P. So every zero-dimensional space has at least one homeomorphism with P.O.T.P. We know ([2], or see [5]) that the unit interval has no expansive homeomorphism. However, as far as the author knows it is unknown whether there is a zero-dimensional space having no expansive homeomorphism. In this section we construct such a space $X$. Note that the space $X$ above is contained in the Cantor set, because the Cantor set is universal for the class of zero-dimensional spaces.

Let $C \subset[0,1]$ be the Cantor set and $S=\{0,1,1 / 2, \cdots\}$ a convergent sequence. Let $X_{n}=\left(C \oplus S^{n}\right) /\left\{0,0_{n}\right\}$ be the quotient space obtained by identifying $\left\{0,0_{n}\right\}$ to a point $x_{n}$, where $0 \in C$ and $0_{n}=(0,0, \cdots, 0) \in S^{n}$, for every $n \in \boldsymbol{N}$, and let $X_{0}=$ $\left\{x_{0}\right\}$ be a one-point space. Let $X=\cup\left\{X_{n}: n \in N \cup\{0\}\right\}$. We give $X$ a topology as follows. Let $\mathscr{B}(x)=\left\{U: U\right.$ is a neighborhood of $x$ in $\left.X_{n}\right\}$ for every $x \in X$, 
$n \in \boldsymbol{N}$, and $\mathscr{B}\left(x_{0}\right)=\left\{\cup\left\{X_{i}: j \leqq i\right\} \cup X_{0}: j \in \boldsymbol{N}\right\}$. Then $\{\mathscr{B}(x): x \in X\}$ is a neighborhood system. Obviously the space $X$ with the topology generated by $\{\mathscr{B}(x)$ : $x \in X\}$ is compact, metrizable and zero-dimensional. Next we show that $X$ has no expansive homeomorphism. To do this let $\varphi$ be a homeomorphism of $X$. The point $x_{n}$ is the only point that has arbitrarily small neighborhoods containing a set homeomorphic to the Cantor set, a set homeomorphic to $S^{n}$, and no set homeomorphic to $S^{n+1}$. Therefore we have $\varphi\left(x_{n}\right)=x_{n}$ for every $n \in \boldsymbol{N}$. Thus $\varphi$ has infinitely many fixed points. Hence $\varphi$ is not expansive.

After I finished writing an early version of this paper, I knew that T. Shimomura [7] also proved Theorem 1 , independently.

\section{References}

[1] Aoki, N., The spliting of zero-dimensional automorphisms and its application, Colloq. Math. 49 (1985), 161-173.

[2] Bryant, B.F., Unstable self-homeomorphisms of a compact space, Vanderbilt University, Thesis, 1954.

[3] Dateyama, M., Homeomorphisms with the pseudo orbit tracing property of the Cantor set, Tokyo J. Math. 6 (1983), 287-290.

[4] Fujii, S., Distal homeomorphisms with the pseudo orbit tracing property and total disconnectedness, Preprint.

[5] Jakobsen, J.F. and Utz, W.R., The nonexistence of expansive homeomorphisms on a closed 2-cell, Pacific J. Math. 10 (1960), 1319-1321.

[6] Sears, M., Expansive self-homeomorphisms of the Cantor set, Math. System Theory 6 (1972), 129-132.

[7] Shimomura, T., The pseudo-orbit tracing property and expansiveness on Cantor set, Preprint.

Institute of mathematics

University of Tsukuba

Tsukuba-shi

Ibaraki 305

Japan 